\title{
EFECTOS NEGATIVOS DEL USO DE BENZODIACEPINAS EN ADULTOS MAYORES: UNA BREVE REVISIÓN
} NEGATIVE EFFECTS OF BENZODIAZEPINE USE IN OLDER ADULTS: A BRIEF REVIEW

\section{CLAUDIO ROJAS-JARA 1, FRANCISCA CALQUIN 1, JAVIERA GONZÁLEZ 1, ENZO SANTANDER 1, MEREDITH VÁSQUEZ 1}

1. UNIVERSIDAD CATÓLICA DEL MAULE, Talca, Chile

\begin{abstract}
Introduction: One part of polypharmacy is related to different medical conditions that come with age in senior patients. Whether they are adequately prescribed or not, most pharmaceutical compounds may potentially produce negative effects in consumers. One of the most highly prescribed psychopharmaceutics in seniors is benzodiazepine. This drug, usually prescribed to treat insomnia or anxiety, is said to be potentially risky for patients' health. Objective: To determine the existence of scientific evidence of benzodiazepine negative effects in seniors. Method: Scientific publications from 2007 through to 2017 were systematically consulted. The database was Scielo, Pubmed, Redalyc, Ebscohost, and Google Scholar. Keywords for the search included seniors, benzodiazepines, physical and psychological effects, psychopharmaceutics, both in English and Spanish. Findings: The most highly consumed benzodiazepine by seniors is Diazepam. Anxiety and insomnia are among the most frequent reasons for its prescription. Regarding negative effects, this drug is highly addictive. Side effects include a high probability of falling, leaving bone fractures as a result, decline of cognitive abilities, and a paradoxical increase of the symptoms for which it was originally prescribed. Senior consumers of benzodiazepine are more frequently female.
\end{abstract}

KEY WORDS: Seniors, benzodiazepines, physical and psychological effects, psychotropic drugs.

\section{CÓMO CITAR / HOW TO CITE}

Rojas-Jara, C., Calquin, F., González, J., Santander, E., \& Vásquez, M. (2019). Efectos negativos del uso de benzodiacepinas en adultos mayores: Una breve revisión. Salud \& Sociedad, 10(1), 40-50. doi: 10.22199/S07187475.2019.0001.00003

\footnotetext{
1. Univer sidad Católica del Maule; Avenida San Miguel 3605, Talca, Chile.0RCID: 0000-0002-1698-6949 E-mail: crojasi@ucm.cl
} 


\section{INTRODUCCIÓN}

En 1984 la Organización Mundial de la Salud estableció el término Adulto Mayor (AM) para hacer referencia a toda persona con edad superior a 60 años (Licas, 2015). En este grupo etário resulta esperable hablar de tratamientos farmacológicos puesto que es una etapa en la cual se presentan diversos cambios y se evidencian múltiples condiciones médicas que conllevan a una polimedicación, y, a su vez, una interacción farmacológica que puede producir efectos secundarios de diversa índole en la persona (Rojas-Jara et al., 2017).

Durante los últimos 20 años se ha presentado un incremento importante en el consumo de los psicofármacos a nivel mundial, siendo las benzodiacepinas (BZD) uno de los fármacos más prescritos en la población $\mathrm{AM}$ en las últimas décadas (Alvarenga, Loyola, Firmo, Lima-Costa, y Uchoa, 2008). Fue Leo Sternback quien descubrió estos fármacos en el año 1930, introduciéndolas a la práctica clínica en 1959 como clordiazepóxido, una BZD ansiolítica conocida como librium (por la terminación de la palabra equilibrium). Sin embargo, no fue sino hasta el año 1963 cuando se comenzó a utilizar en gran escala con la aparición del diazepam (Valium), gracias a su eficacia terapéutica por sobre las sustancias utilizadas hasta el momento como ansiolíticos, convirtiéndose rápidamente en los fármacos más utilizados reemplazando así a los barbitúricos (López, Aroche, Bestard, y Ocaña, 2010; Rosas-Gutiérrez, Simón-Arceo, y Mercado, 2013).

En América Latina existe un potencial crecimiento de la población de AM, por lo que el uso racional que existe con relación a la prescripción de psicofármacos es de real importancia (Gómez, León, Macuer, Alves, y Ruiz, 2017). Debido a esto, conocer los efectos provocados por las BZD más allá de los farmacológicos es cada vez más relevante. Algunos efectos conocidos provocados por su uso en AM son: deterioro cognitivo (Florián y Buitrago, 2013; Islam et al., 2016), rendimiento psicomotor (Danza et al., 2015; Uhart et al., 2012), dependencia (Nkogho Mengue, Abdous, Berbiche, Préville, y Voyer, 2013; Minaya, Ugalde, y Fresán, 2009), entre otras. El primer acercamiento a las BZD suele ser por prescripción médica, a diferencias de otras drogas sean legales o ilegales. De igual forma, el uso prolongado de BZD se ha asociado al desarrollo de dependencia, aun cuando sea en dosis terapéuticas (Nkogho Mengue et al., 2013).

Aparece relevante desarrollar este análisis pues los adultos mayores, según diversos autores, presentan una alta prevalencia en el consumo de BZD (Alvarenga, et al., 2008; Calero, Oropesa, González, Roca, y Blanco, 2009). Además, a pesar de que su uso inicial suele ser bajo prescripción médica, no siempre ocurre de esta manera (Passos et al., 2016), por lo que conocer los efectos tanto físicos y psicológicos del consumo y cómo ellos pueden llegar a influir en la calidad de vida de los adultos mayores se transforma en un imperativo.

A partir de estos antecedentes nuestra revisión se orienta a determinar si existe evidencia científica actual acerca de los efectos físicos y psicológicos negativos asociados al uso de BZD en AM.

\section{MÉTODO}

Se realizó una revisión sistemática para analizar las publicaciones científicas entre el periodo 2007-2017, relacionada con el uso de BZD en AM. Los artículos fueron obtenidos en las bases de datos Scielo, Pubmed, Redalyc, Ebscohost y Google Scholar. Las palabras claves a utilizar fueron: adulto mayor, benzodiacepinas, efectos físicos y psicológicos, psicofármacos. Posteriormente se realizó la búsqueda de estos términos en idioma inglés.

Se excluyeron los artículos científicos que no contenían información relevante al tema central de investigación, aquellos no dirigidos 
o enfocados en $A M$, otros que no pertenecían a las bases de datos establecidas para fines de esta investigación, como también artículos con una antigüedad mayor a 10 años.

El proceso de selección de artículos se dividió en las siguientes etapas: (1) estrategia de búsqueda bibliográfica, (2) selección de artículos según los criterios de exclusión e inclusión, (3) creación de fichas bibliográficas durante la búsqueda con el fin de evitar duplicados, (4) extracción de información relevante de los estudios encontrados, (5) análisis crítico y categorización de la información, orientandose bajo el método PRISMA.

En total la revisión utilizó 44 artículos científicos cuyos principales documentos, elementos y análisis se exponen a continuación.

Antecedentes

Las BZD corresponden a una familia de fármacos que se caracterizan por ser depresores del sistema nervioso central, con efectos miorelajantes (relajación muscular), anticonvulsivantes, ansiolíticos e hipnóticos. Su mecanismo de acción está asociado al complejo de receptores gamma-amino butírico (GABA), los receptores inhibitorios más grandes del sistema nervioso central. Las moléculas benzodiacepínicas se unen a estos receptores que constan de un canal iónico y varias sub-unidades que se fijan a otras sustancias (alcohol, BZD y algunos barbitúricos). Los receptores GABA son los encargados de modular la polarización de las neuronas mediante el cierre o la apertura de los canales de cloruro. Su clasificación está determinada por el tiempo de vida media de cada tipo de BZD (larga, mayor a 24 horas, e.g. diazepam; media, de 6 a 24 horas, e.g. lorazepam, alprazolam, bromazepam; corta, menor a 6 horas, e.g. midazolam, triazolam) relacionado al tiempo de eliminación de cada fármaco. Esto es importante considerar ya que estaría relacionado con los posibles efectos adversos que puedan causar en quien la use (Alvarenga et al., 2008; Florián y Buitrago, 2013; Riquelme, 2008; Núñez, Núñez, Gómez, y Núñez, 2012).

Las benzodiacepinas son utilizadas frecuentemente para el insomnio, tratamiento de la ansiedad, trastornos convulsivos, síndrome de abstinencia al alcohol, anestesia, cirugía menor $y$ enfermedades neuromusculares (Honorato, Azanza, y Cuena, 2016). Estos medicamentos son poco recomendados en adultos mayores debido a los cambios fisiológicos y farmacológicos que este grupo etario experimenta, asociándose su uso crónico incluso con bajas puntuaciones en escalas de valoración cognitiva (GarcíaBaztán et al., 2014). Esto se debe a que su uso prolongado genera un aumento en las dosis necesarias (o tolerancia) para poder lograr los efectos esperados, especialmente en los hipnóticos o anticonvulsivos (Cucco, 2008).

\section{Prescripción de benzodiacepinas}

En este punto es importante establecer la diferencia entre la prescripción apropiada y aquella que suele ser inapropiada. Para la Organización Mundial de la Salud el uso adecuado de fármacos implica que los pacientes reciban medicamentos acordes a sus necesidades clínicas, en dosis y tiempos adecuados a las particularidades de su situación, suponiendo el mínimo costo para ellos y la comunidad (Rosas-Gutiérrez, Simón-Arceo, y Mercado, 2013). Como en todo ámbito se generan discusiones acerca de qué tan necesario puede ser un fármaco o cuál es la evaluación que se hace acerca de riesgo versus beneficio de este. Se considera que dentro de la población adulto mayor sólo un tercio de las prescripciones de benzodiacepinas son apropiadas, dejando ver que más de la mitad de las prescripciones resultan inapropiadas (Barberá et al., 2008).

Un estudio realizado de Sánchez y Hernández (2010) establece que las benzodiacepinas se consumen por dos motivos principales: por prescripción médica 
o por automedicación. Estos fármacos son prescritos para diversos cuadros sintomáticos, entre los cuales se encuentran: trastornos de ansiedad generalizada, trastorno por estrés postraumático, trastorno de pánico, trastorno adaptativo con ánimo ansioso, reacciones agudas al estrés e insomnio (Ayuso, 2008). Alvarenga, Giacomin, Loyola, Uchoa y Oliveira (2014) señalan que las prescripciones más frecuentes son realizadas por preocupación, nerviosismo y dificultad para dormir.

Otra investigación realizada con población AM perteneciente a Corea en el periodo 2009-2013, señala que estos son quienes presentan un mayor consumo de BZD con relación a otros países. Según muestran sus resultados, la prescripción en esta población se realiza en bajas dosis, pero durante un tiempo prolongado. A lo largo del estudio no se mostraron mayores cambios en la forma de recetar esta droga, sin embargo se dio cuenta de un aumento de esta práctica en población considerada vulnerable (Hwang et al., 2017).

Dentro del grupo etario de los AM la prescripción de BZD se asocia a cuadros de insomnio y ansiedad (Calero et al., 2009). Un estudio realizado en Argentina refiere que la alta prescripción no se limita al insomnio y ansiedad sino que también es utilizada como un medicamento complementario para problemas no necesariamente psiquiátricos, como por ejemplo, el tratamiento de colon irritable (Agostini-Zampieron, Aybar-Maino, Heit-Barbini, Arca-Fabre, y Mónaco-Ditieri, 2015).

Según Bartlett, Abrahamowicz, Grad, Sylvestre y Tamblyn (2009) entre los predictores más comunes para la indicación de BZD en AM se incluyen: a) la cantidad de médicos que prescriben, b) ser mujer, c) tener diagnóstico de depresión, d) abuso 0 dependencia de alcohol y otras drogas, e) medicamentos en general, siendo los antidepresivos el pronosticador más fuerte. Por cada médico visitado la probabilidad de prescripción de BZD aumenta un 12\%. Por el contrario, predictores que bajan la probabilidad de recibir una indicación de BZD en AM son el aumento de la edad, un mayor número de estadías en hospitales de cuidados intensivos, tener una lesión por una caída que requirió atención médica, un diagnóstico de accidente cerebrovascular y trastornos convulsivos.

\section{Consumo de benzodiacepinas por sexo}

Silva, Vergara, Yera y Freijoso (en Sánchez y Hernández, 2010) en un estudio realizado en 81 policlínicos pertenecientes a la ciudad de la Habana, en Cuba constataron que un $73,6 \%$ de los consumidores de benzodiacepinas corresponden al sexo femenino, con un predominio en las mujeres mayores a 60 años. Asimismo, el diazepam fue la BZD que más se consumió en ambos sexos, pero por motivos diferentes. En el caso masculino por indicación facultativa, mientras que en el sexo femenino por automedicación y una alta prescripción por insomnio y ansiedad (Sánchez y Hernández, 2010). Bajo la misma línea, existe otro estudio efectuado en el año 2008 que considera un mayor consumo de BZD en el sexo femenino, no obstante, cabe destacar que la muestra estaba constituida en su mayoría por mujeres con un $82 \%$, a diferencia de los hombres representada por un $18 \%$ (Riquelme, 2008). En coincidencia otros autores también dan cuenta de un mayor consumo de BZD en mujeres (Passos et al., 2016; Rossat et al., 2011; Velert, Velert, Salar, Avellana, y Moreno, 2012).

Efectos negativos asociados al uso de Benzodiacepinas

Un estudio plantea que las BZD corresponden a los psicofármacos más utilizados en el AM y que, por ende, su uso conlleva una alta presentación de diversos efectos adversos (Flores, 2016). Airagnes, Pelissolo, Lavallée, Flament y Limosín (2016) señalan que estos efectos suelen generarse debido a situaciones consideradas como inapropiadas en el tratamiento con BZD, un ejemplo de ello es 
la alta prescripción de este fármaco y una duración excesivamente prolongada de su uso. Otro factor que puede provocar efectos adversos es el uso en contextos donde estaría contraindicado, como el uso de BZD de acción prolongada en adultos mayores. Agregan además estos autores que a pesar de las actualizaciones respecto a su utilización muchos médicos siguen promoviendo su prescripción de manera desmedida.

Dentro de los diversos efectos negativos que emergen de un uso inapropiado de BZD (sea por errores en la prescripción o la automedicación) se encuentran la sedación, ataxia, vértigo, descoordinación motora y mayor riesgo de caídas, como también la amnesia global transitoria, déficit en el procesamiento sensorial y en el razonamiento verbal (Ayuso, 2008; Velert, Moreno, Velert, y Salar, 2012). Schneemilch y Brinkers (2015) por su parte, plantean que en el uso a corto plazo de BZD se evidencian síntomas como mareos, dolores de cabeza, náuseas y taquicardia y en el uso a largo plazo de se asocian con ansiedad, fatiga, sueño y estados del ánimo irritables. Con relación a lo anterior, Mato (en Flores, 2016) señala que una prescripción inadecuada de BZD puede producir diversos efectos adversos como somnolencia, mareos 0 confusión, trastornos motores, incremento en el riesgo de sufrir caídas y deterioro funcional.

La demencia es definida como el deterioro progresivo de habilidades intelectuales como la memoria. Esta enfermedad tiene tres características principales: es permanente, se adquiere y afecta funciones del dominio intelectual de manera progresiva en el tiempo (Florián y Buitrago, 2013). En cuanto a su relación con el consumo de BZD, se ha encontrado que quienes las consumen tienen un $78 \%$ más de probabilidades de padecer demencia, aunque no se puede establecer que sea un factor directo (Islam et al., 2016). Lo mismo plantean Billioti de Gage, Pariente, y Bégaud
(2015) quienes señalan que, si bien en 8 estudios por ellos revisados se establece una relación entre el uso de BZD y el riesgo de demencia, existen factores como los criterios diagnósticos utilizados en los estudios y la falta de control en varios de ellos (como el consumo de alcohol, ansiedad, depresión, insomnio y nivel educativo) que impiden establecer una causalidad directa y segura entre ambos. También Pomara et al. (2015) señalan que la memoria y el rendimiento psicomotor se ven afectados de diversas maneras tras consumir Lorazepam, dependiendo del tiempo transcurrido desde el consumo. Velert et al. (2012) plantean que quienes disminuyen el consumo de estos fármacos presentan menor deterioro cognitivo y reacciones adversas que quienes no lo hacen. Florián y Buitrago (2013), además concluyen que mientras menor sea el tiempo de no consumo de BZD, o abstinencia, puede haber un incremento en el riesgo de padecer demencia. Otra investigación señala que si bien, existe una relación entre el uso de estos fármacos y deterioro cognitivo, no es significativa. Además concluye que los usuarios de BZD tienen una mayor prevalencia de sintomatología depresiva y más probabilidades de reportar el consumo de tabaco y alcohol (Zhang, Zhou, Meranus, Wang, y Kukull, 2016).

En cuanto a la ansiedad se encontró una relación significativa que aumentaba con el uso más frecuente de BZD, y el uso crónico de estas se asoció con un triple riesgo de ansiedad durante el seguimiento. La satisfacción de vida, por otro lado, disminuyó al existir uso de BZD, siendo el uso crónico de estas el factor más determinante. Además se presentó una mayor dificultad para conciliar el sueño en AM al consumir BZD, y durante el seguimiento se relaciona en mayor medida con el uso intermitente y el crónico. En consideración a la salud general percibida y uso de BZD se presentó una relación relativamente débil (Nordfjærn, 2013). Por otro lado, se ha demostrado que el uso de BZD de al menos 3 meses puede 
ser suficiente para tener un efecto nocivo sobre el estado de ánimo y/o ansiedad del AM. Incluso los problemas psicomotores como pérdida del equilibrio y reducción de la motricidad, podrían afectar la ansiedad del $A M$, ya que pueden producirse lesiones 0 accidentes automovilísticos. De igual manera, algunos problemas de memoria 0 atención pueden ser confundidos con demencia senil provocando cierto malestar en los AM (Cook, Marshall, Masci, y Coyne, 2007 en Rouleau, Bélanger, O'Connor, y Gagnon, 2011).

Sobre los efectos conductuales del uso de BZD, uno particularmente común es la dependencia. Esta es definida por Aguirre y Sifri (2016) como un trastorno conductual en donde los efectos biológicos causados por determinadas sustancias producen una falta de control sobre el consumo de ellas. Tanto las BZD de vida media corta como las de vida media larga presentan un alto potencial adictivo (Schneemilch y Brinkers, 2015). Minaya, Ugalde y Fresán (2009) señalan que aun cuando estos medicamentos son suministrados en dosis terapéuticas por un tiempo superior a 4 semanas se asocia a la posibilidad de desarrollar dependencia. Por otro lado, Nkogho Mengue et al. (2013) plantean que los usuarios de BZD que presentan mayor riesgo de desarrollar dependencia son mujeres y AM que padecen al menos cinco enfermedades crónicas. Estos últimos, al presentar dependencia, son más propensos a consultar a un médico especialista. Lader (2014) señala que, si bien la retirada de muchos medicamentos puede estar acompañada de problemas, particularmente en cuanto a las BZD la mayor dificultad se encuentra asociada a su efecto hipnótico, teniendo la mayor recurrencia de síntomas que en un principio fueron paliados con estos fármacos, como el insomnio. Esto provoca un circuito recursivo donde al presentar nuevamente el síntoma el usuario vuelve a consumir el medicamento sin prescripción médica. Olivera (en Flores, 2016) explica que el mecanismo de acción sedante y/o hipnótico que tienen las BZD se ubican en el receptor gamma aminobutírico (GABA), como el primer depresor endógeno del sistema nervioso central. Se cree que el uso crónico de las benzodiacepinas, puede llevar a una regulación negativa del receptor GABA-inhibidor y cambios en el complejo receptor-agonista lo que genera una baja de la sensibilidad al agonista. Todos estos cambios explican de alguna manera los efectos adversos de dependencia, tolerancia y síndrome de abstinencia relacionados al consumo prolongado de benzodiacepinas.

\section{Efectos colaterales del uso de benzodiacepinas}

En cuanto a los efectos físicos colaterales producidos por el consumo de BZD se ha asociado su prescripción a accidentes de tráfico en carretera, caídas y fractura de cadera, donde han demostrado que quienes consumen BZD son más propensos a participar de alguno de estos episodios, debido a lo afectadas que se ven habilidades cognitivas complejas como la conducción y la coordinación motriz (Airagnes et al., 2016; Flores, 2016; Formiga et al., 2012; Lader, 2014). Por otro lado, se encontró que son las mujeres de edad avanzada quienes sufren mayores efectos físicos colaterales por el uso de estos medicamentos, siendo el bromazepam la BZD más consumida e influyente en ello, aumentando considerablemente el porcentaje de caídas recurrentes y lesiones por caídas (Rossat et al., 2011; Uhart et al., 2012; Danza et al., 2015; Softic, Beganlic, Pranjic, y Sulejmanovic, 2013). Contrario al estudio anterior, una investigación realizada en Canadá (Sylvestre, Abrahamowicz, Čapek, y Tamblyn, 2011) no encontró asociaciones significativas entre el riesgo de sufrir lesiones por caídas y el clordiazepóxido, lorazepam y bromazepam, a pesar de ser lorazepam la BZD más consumida. Sin embargo, en este mismo estudio, se posiciona el flurazepam como la BZD más significativa en términos de relaciones con caídas y lesiones por ellas, seguido por el alprazolam (27\%) y el clonazepam (20\%), mientras que en el caso del temazepam el riesgo de caídas disminuye en consumidores habituales, 
teniendo más posibilidades de sufrir caídas si el medicamento es retirado.

Adicionalmente, una revisión sistemática reciente señala que un factor de riesgo relacionado con el consumo de BZD y las caídas sería la cantidad de tiempo que estas son ingeridas. Por este motivo se recomienda que los médicos no prescriban BZD por periodos prolongados, especialmente si los pacientes presentan una edad avanzada y no existiera una supervisión cercana. Otro factor está relacionado con la dosificación ya que se vincularía a un mayor riesgo de caídas, donde se ha comprobado que altas dosis de BZD presentes en el sistema nervioso central estarían asociadas a un aumento en la posibilidad de alguna caída (Díaz-Gutiérrez et al., 2017).

En un estudio en el cual se midieron parámetros respiratorios como volumen tidal (cantidad de aire que es desplazado a lo largo de la inhalación y exhalación normal), frecuencia respiratoria y volumen respiratorio (volumen de aire inhalado y exhalado por una persona por minuto) luego de la administración pre-operatoria de BZD de manera analgésica, se encontró que tanto volumen tidal como volumen respiratorio disminuyeron de manera significativa, demostrando así una respuesta potencialmente peligrosa (Gonzalez, Mehta, Brayanov, y Mullen, 2017).

\section{CONCLUSIONES}

Con base al análisis y hallazgos encontrados en esta revisión, se puede destacar inicialmente que las BZD son fármacos ampliamente utilizados para el tratamiento de síntomas relacionados con la ansiedad y el insomnio. Según los estudios revisados, la más investigada es el diazepam, seguida por el bromazepam.

Dentro de esta práctica, y según la evidencia encontrada, existe una prescripción importante de BZD hacia los AM pudiendo resultar riesgosa para su salud.
El consumo de BZD presenta una mayor prevalencia en el sexo femenino, no obstante, los estudios revisados estaban conformados en su mayoría por mujeres AM, situación que podría haber influido y sesgado los resultados obtenidos a partir de ellos.

En cuanto a los efectos negativos generados por el consumo de BZD, y para efectos netamente de estructura de esta revisión, se separaron en psicológicos y físicos. Dentro de los primeros se subdividió en efectos cognitivos y conductuales. En los efectos cognitivos se encuentran el deterioro cognitivo y demencia, en los cuales no existe un consenso, ya que se observó en algunos estudios la existencia de una relación significativa entre el consumo de BZD y una disminución de las capacidades cognitivas, mientras otras investigaciones señalan que no se puede asegurar que exista una causalidad directa entre el uso de este psicofármaco y los efectos mencionados a pesar de haberse encontrado una relación entre ellos. Un aspecto psicológico que logra un mayor consenso es la ansiedad, la cual se ve afectada de manera negativa por el consumo de BZD ya que su aumento sería significativo, llegando a incrementar hasta tres veces su complejidad si éstas son consumidas de manera crónica, aun cuando se utilicen -paradójicamente- estos fármacos para el tratamiento de la misma (Nordfjærn, 2013). Con respecto a los efectos conductuales y el uso de BZD el más recurrente dentro de la evidencia revisada es la posibilidad de desarrollar algún grado de dependencia, inclusive cuando sean administradas en dosis terapéuticas. Dentro de los efectos físicos colaterales asociados con el consumo de BZD en AM se encontró una relación significativa con el riesgo de sufrir caídas y sus secuelas (como por ejemplo fractura de cadera), sin embargo, en ello tampoco existe necesariamente un consenso en las investigaciones.

La revisión desarrollada da cuenta de que esta temática es emergente en distintos países y continentes donde el consumo de BZD no resulta algo ajeno, teniendo en 
cuenta las diferencias que podrían existir a nivel cultural. Los estudios abordaban de manera similar el consumo de BZD dando cuenta en la mayoría de aspectos como un uso inapropiado, riesgo de dependencia y un posible riesgo de ver deteriorada la salud de los AM que las usan.

En coincidencia con la evidencia revisada, y las propias limitaciones de nuestro estudio, consideramos esencial la realización de nuevas investigaciones que ofrezcan resultados más precisos para afirmar o descartar de manera fiable y generalizable el tipo de efectos que podría tener el uso de BZD en AM, los riesgos asociados, la diferenciación de consumo por tipo de BZD, el nivel educativo de los participantes y el tiempo de consumo, para así evitar afirmaciones vagas y producir evidencia que favorezca acciones de salud en directo beneficio de la población que transita por este rango etario. Resulta importante la divulgación y educación a los consumidores, médicos y población en general sobre el uso de BZD, en particular en la $A M$, con la finalidad de que se lleve a cabo un uso responsable y efectivamente terapéutico, evitando así la iatrogenia.

\section{REFERENCIAS}

Agostini-Zampieron, M., Aybar-Maino, J., Heit-Barbini, F., Arca-Fabre, A., y Mónaco-Ditieri, M. (2015). Uso de psicofármacos en un grupo de adultos mayores de Rosario, Argentina. Atención Familiar, 22, 10-13. doi: 10.1016/S1405-8871(16)30038-4

Aguirre, M., y Sifri, C. (2016). Paciente femenino de tercera edad, autovalente, adicción a benzodiacepinas. Psiquiatría y Salud Mental, 33, 51-56.

Airagnes, G., Pelissolo, A., Lavallée, M., Flament, M., y Limosin, F. (2016). Benzodiazepine misuse in the elderly: risk factors, consequences, and management. Current Psychiatry Reports, 18(10), $89 . \quad$ doi: 10.1007/s11920-016-0727-9
Alvarenga, J., Giacomin, K., Loyola, A., Uchoa, E., y Oliveira, J. (2014). Uso crônico de benzodiazepínicos entre idosos. Revista de Saúde Pública, 48(6). doi:10.1590/S00348910.2014048004986

Alvarenga, J., Loyola, A., Firmo, J., LimaCosta, M., y Uchoa, E. (2008). Prevalence and sociodemographic characteristics associated with benzodiazepines use among community dwelling older adults: the Bambuí Health and Aging Study (BHAS). Revista Brasileira de Psiquiatria, 30(1), 7-11. doi: 10.1590/S151644462006005000062

Ayuso, J. L. (2008). ¿Está justificado el tratamiento prolongado con benzodiacepinas? Salud Mental, 31(6), 429-430.

Barberá, T., Avellana, J., Codoñer, P., Bou, R., Sanahuja, M., y Moreno, L. (2008). Prescripción inapropiada de benzodiazepinas en la población mayor de la comunidad valenciana. Pharmaceutical Care España, 10(1), 27.

Bartlett, G., Abrahamowicz, M., Grad, R., Sylvestre, M., y Tamblyn, R. (2009). Association between risk factors for injurious falls and new benzodiazepine prescribing in elderly persons. BMC Family Practice, 10(1). doi: 10.1186/1471-2296-10-1

Billioti de Gage, S., Pariente, A., y Bégaud, B. (2015). Is there really a link between benzodiazepine use and the risk of dementia?. Expert Opinion on Drug Safety, 14(5), 733-747. doi: 10.1517/14740338.2015.1014796

Calero, L., Oropesa, D., González, M., Roca, A., y Blanco, K. (2009). La prescripción de benzodiacepinas en el adulto mayor, un problema médico social actual. Correo Científico Médico de Holguín, 13(2).

Cucco, L. (2008). ¿Hacemos un uso adecuado de las benzodiacepinas? Conocimientos prácticos para atención primaria. Revista de Medicina General, 
112, 780-7.

Danza, A., Rodríguez, M., López, M., Agorio, D., Caleri, A., Patiño, I., Casal, M., y Díaz, L. (2015). Benzodiazepinas y fractura de cadera: estudio de casos y controles. Revista Médica del Uruguay, 31(2), 120-127.

Díaz-Gutiérrez, M., MartínezCengotitabengoa, M., Sáez, E., Cano, A., Martínez-Cengotitabengoa, M., Besga, A., Segarra. R., y GonzálezPinto, A. (2017). Relationship between the use of benzodiazepines and falls in older adults: a systematic review. Maturitas, 101, 17-22. doi: 10.1016/j.maturitas.2017.04.002

Florián, J., y Buitrago, Y. (2013). Uso de benzodiacepinas y demencia: una revisión sistemática de la literatura. (Tesis de maestría). Universidad del Rosario, Bogotá - Colombia.

Flores, C. (2016). Efectos adversos de las benzodiacepinas en el adulto mayor de la UMF 16 Querétaro (Doctoral dissertation).

Formiga, F., Duaso, E., Ruiz, D., San José, A., Urrutia, A., y López-Soto, A. (2012). Utilización de benzodiacepinas según el motivo de ingreso. Medicina Clínica, 139(12), 531-534. doi: 10.1016/j.medcli.2012.05.037

García-Baztán, A., Roqueta, C., MartínezFernández, M. I., Colprim, D., Puertas, P., y Miralles, R. (2014). Prescripción de benzodiacepinas en el anciano en diferentes niveles asistenciales: características y factores relacionados. Revista Española de Geriatría y Gerontología, 49(1), 24-28. doi: 10.1016/j.regg.2013.04.001

Gómez, S., León, T., Macuer, M., Alves, M., y Ruiz, S. (2017). Uso de benzodiazepinas en adultos mayores en América Latina. Revista Médica de Chile, 145(3), 351-359. doi: 10.4067/S0034-98872017000300009

Gonzalez, L., Mehta, J., Brayanov, J., y Mullen, G. (2017). Quantification of respiratory depression during preoperative administration of midazolam using a non-invasive respiratory volume monitor. Plos One, 12(2). doi: 10.1371/journal.pone.0172750

Honorato, J., Azanza, J., y Cuena, R. (2016). Farmacología clínica de las benzodiacepinas. Revista de Medicina de la Universidad de Navarra, 26(4), 21.

Hwang, H., Han, S., Choi, H., Park, C., Kim, M., y Kim, H. (2017). Trends in the prescription of benzodiazepines for the elderly in Korea. BMC psychiatry, 17(1), 303. doi: 10.1186/s12888-017-1467-z

Islam, M., Iqbal, U., Walther, B., Atique, S., Dubey, N., Nguyen, P., Poly, T., Masud, J., Li, Y., y Shabbir, S. (2016). Benzodiazepine use and risk of dementia in the elderly population: a systematic review and meta-analysis. Neuroepidemiology, 47(3-4), 181-191. doi: $10.1159 / 000454881$

Lader, M. (2014). Benzodiazepine harm: how can it be reduced?. British Journal of Clinical Pharmacology, 77(2), 295-301. doi: 10.1111/j.1365-2125.2012.04418.x

Licas, P. (2015). Nivel de depresión del adulto mayor en un centro de atención integral SJM-VMT 2014. (Tesis de pregrado). Universidad Nacional mayor de San Marcos, Perú.

López, A., Aroche, A., Bestard, J., y Ocaña, N. (2010). Uso y abuso de las benzodiazepinas. Medisan, 14(4), 0-0.

Minaya O., Ugalde, O., y Fresán, A. (2009). Uso inapropiado de fármacos de prescripción: dependencia a benzodiazepinas en adultos mayores. Salud Mental, 32(5), 405-411.

Nkogho Mengue, P., Abdous, B., Berbiche, D., Préville, M., y Voyer, P. (2013). Impact benzodiazepine dependence on the use of health services: senior health study. Geriatrie et Psychologie Neuropsychiatrie $d u$ Vieillissement, 11(3), 229-236. doi: 10.1684/pnv.2013.0411

Nordfjærn, T. (2013). Prospective associations between benzodiazepine use and later life satisfaction, somatic pain and psychological health among the elderly. Human 
Psychopharmacology: Clinical \& Experimental, 28(3), 248-257. doi: 10.1002/hup.2316

Núñez, M., Núñez, P., Gómez, M., y Núñez, M. (2012). Consumo de benzodiacepinas en el adulto mayor. $\mathrm{X}$ Seminario Internacional de Atención Primaria de Salud-Versión Virtual.

Passos, C., Lima, A., Bezerra, F., de Sousa, E., Lopes, C., y de Oliveira, M. (2016). Consumption of benzodiazepínicos between elderly in the strategy family health: integrative review. Revista de Enfermagem UFPE on line, 10(12), 4646-56. doi: 10.5205/1981-8963v10i12a11534p4646-4656-2016

Pomara, N., Lee, S. H., Bruno, D., Silber, T., Greenblatt, D. J., Petkova, E., y Sidtis, J. J. (2015). Adverse performance effects of acute lorazepam administration in elderly long-term users: pharmacokinetic and clinical predictors. Progress in NeuroPsychopharmacology and Biological Psychiatry, 56, 129-135. doi: 10.1016/j.pnpbp.2014.08.014

Riquelme, P. (2008). Uso de benzodiacepinas y su relación con la incidencia de reacciones adversas en pacientes adultos mayores de tratamiento crónico ambulatorio en establecimientos dependientes del sistema de salud de Valdivia. (Tesis de grado Químico Farmacéutico). Universidad Austral de Chile. Valdivia.

Rojas-Jara, C., Ramírez, L., Pinto, C., Concha, C., Chandía, S., Cerda, B., \& Apablaza, B. (2017). Polifarmacia en el adulto mayor y el significado del uso de drogas en la vejez. En I. Fergusson \& C. Rojas (Eds.) Gerontología: actualizaciones y temas emergentes (pp. 137-157). Talca: Universidad Católica del Maule.

Rosas-Gutiérrez, I., Simón-Arceo, K., y Mercado, F. (2013). Mecanismo celular y molecular de la adicción a benzodiacepinas. Salud Mental, 36(4), 325-329.

Rossat, A., Fantino, B., Bongue, B., Colvez,
A., Nitenberg, C., Annweiler, C., y Beauchet, O. (2011). Association between benzodiazepines and recurrent falls: a cross-sectional elderly population-based study. The Journal of Nutrition, Health \& Aging, 15(1), 72-77. doi: 10.1007/s12603-011-0015-7

Rouleau, A., Bélanger, C., O'Connor, K., y Gagnon, C. (2011). Évaluation de l'usage à risque des benzodiazépines chez les personnes âgées: facteurs de risque et impacts. Santé Mentale au Québec, 36(2), 123-44. doi: 10.7202/1008593ar

Sánchez, L., y Hernández, F. (2010). Consumo de benzodiazepinas en pacientes geriátricos del Consultorio \#12, Policlínico "Campo Florido". Revista Cubana de Farmacia, 44(3), 346-353.

Schneemilch, C., y Brinkers, M. (2015). Der suchtkranke Patient in der Anästhesie Patienten mit

Benzodiazepinabhängigkeit.

Anästhesiol Intensivmed Notfallmed Schmerzther, 50(6), 416-424. doi: 10.1055/s-0041-101788

Softic, A., Beganlic, A., Pranjic, N., y Sulejmanovic, S. (2013). The influence of the use of benzodiazepines in the frequency falls in the elderly. Medical Archives, 67(4), 256-259. doi: 10.5455/medarh.2013.67.256-259

Sylvestre, M., Abrahamowicz, M., Čapek, R., y Tamblyn, R. (2011). Assessing the cumulative effects of exposure to selected benzodiazepines on the risk of fall-related injuries in the elderly. International Psychogeriatrics, 24(4), 577-586.

doi: $10.1017 / \mathrm{S} 1041610211002031$

Uhart, M., Odouard, E., Carlier, C., Maire, P., Ducher, M., y Bourguignon, L. (2012). Relationship between benzodiazepines use and falls in the elderly: a multicenter study in three geriatric centers of a university hospital. Annales Pharmaceutiques Francaises, 70(1), 4652. doi: 10.1016/j.pharma.2011.10.002 Velert, J., Moreno, L., Velert, M., y Salar, L. 
(2012). Se puede mejorar el uso de las benzodiacepinas desde la farmacia. Pharmaceutical Care España, 14(3), 94. Velert, J., Velert, M., Salar, L., Avellana, J., y Moreno, L. (2012). Adecuación de la utilización de benzodiacepinas en ancianos desde la oficina de farmacia: un estudio de colaboración médicofarmacéutico. Atención Primaria, 44(7), 402-410. doi: 10.1016/j.aprim.2011.07.018

Zhang, Y., Zhou, X. H., Meranus, D. H., Wang, L., y Kukull, W. A. (2016). Benzodiazepine use and cognitive decline in elderly with normal cognition.

\section{Alzheimer Disease and Associated \\ Disorders, 30(2), 113-117. doi: 10.1097/WAD.0000000000000099}

Todos los trabajos publicados en

Revista Salud \& Sociedad (ISSNe:0718-7475)

están sujetos a una licencia Creative Commons

Reconocimiento 4.0 Internacional

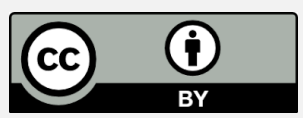

\section{RESUMO}

Introdução: 0 uso de múltiplos medicamentos tem relação com diferentes condições médicas que surgem naturalmente em adultos. Nos múltipos psicofármacos, que são ou não devidamente prescritos, é possivel encontrar potenciais efeitos negativos para seus consumidores. Um dos psicofarmacos de maior prescrição para adultos é o benzodiazepínico, indicado regularmente para a insonia ou ansiedade, sobre 0 qual existem referencias que assinalam que 0 uso desse tipo de medicamento produz um potencial risco para a saúde. Objetivo: determinar a existência de evidência científica atual sobre os efeitos negativos do uso de benzodiazepínicos em adultos. Método: foi realizada uma revisão sistemática de publicaçnoes científicas entre 2007-2017 nas bases de dados Scielo, Pubmed, Redalyc, Ebscohost y Google Scholar. As palavras chaves utilizadas foram: adulto mayor, benzodiacepinas, efectos físicos y psicológicos, psicofármacos tanto en castellano como em inglés. Resultados: 0 benzobiazepínico mais consumido por adultos é o diazepam. Os motivos de prescrição mas frequente snao ansiedade e insonia. No que se refere aos efeitos negativos associados foi encontrado um alto risco de desenvolvimento de dependência, assi m como certos efeitos colaterais de seu uso, onde se destaca uma maior probabilidade de quedas e consequências físicas associadas a elas (ex. Fraturas), deterioração cognitiva, e acentuação paradóxica dos sintomas para os quais são prescritos inicialmente esses medicamentos. Os consumidores mais frequentes de benzodiazepínicos são adultos do sexo feminino.

PALAVRAS-CHAVE: Adultos, benzodiazepínicos, consequências físicas e psicológicas, psicofármacos.

\section{RESUMEN}

Introducción: la polifarmacia tiene relación con diferentes condiciones médicas que surgen naturalmente en adultos mayores. En los múltiples preparados farmacológicos, que son o no debidamente prescritos, es posible encontrar potenciales efectos negativos para sus consumidores. Uno de los psicofármacos de mayor prescripción en adultos mayores es la benzodiacepina, indicado regularmente para el insomnio o la ansiedad, sobre el cual existen referencias que señalan que el uso de este tipo de fármacos denota un potencial riesgo a su salud. Objetivo: determinar la existencia de evidencia científica actual sobre de los efectos negativos del uso de benzodiacepinas en adultos mayores. Método: se realizó una revisión sistemática de publicaciones científicas entre 2007-2017 en las bases de datos Scielo, Pubmed, Redalyc, Ebscohost y Google Scholar. Las palabras claves a utilizar fueron: adulto mayor, benzodiacepinas, efectos físicos y psicológicos, psicofármacos tanto en castellano como en inglés. Resultados: la benzodiacepina más consumida por adultos mayores es el diazepam. Los motivos de prescripción más frecuente son ansiedad e insomnio. En cuanto a los efectos negativos asociados se encontró un alto riesgo para desarrollar dependencia, como también ciertos efectos colaterales de su uso donde se destaca una mayor probabilidad de caídas y consecuencias físicas asociadas a ellas (e.g. fracturas), deterioro cognitivo y acentuación paradóiica de los síntomas para los cuales son prescritos inicialmente estos medicamentos. Consumidores más frecuentes de benzodiacepinas en adultos mayores son de sexo femenino.

PALABRAS CLAVE: Adulto mayor, benzodiacepinas, efectos físicos y psicológicos, psicofármacos. 